\title{
Cuticle Matrix Imaging by Histochemistry, Fluorescence, and Electron Microscopy
}

\author{
Nada Žnidaršič ${ }^{1 *}$, Polona Mrak ${ }^{1}$, Eva Rajh ${ }^{2}$, Kristina Žagar Soderžnik ${ }^{3}$, Miran $\check{C ̌}^{3}$ and Jasna Štrus $^{1}$ \\ ${ }^{1}$ Department of Biology, Biotechnical Faculty, University of Ljubljana, Večna pot 111, 1000 Ljubljana, Slovenia \\ ${ }^{2}$ Faculty of Chemistry and Chemical Technology, University of Ljubljana, Večna pot 113, 1000 Ljubljana, Slovenia \\ ${ }^{3}$ Department for Nanostructured Materials, Jožef Stefan Institute, Jamova cesta 39, 1000 Ljubljana, Slovenia
}

Received: 10 October 2017; accepted: 05 March 2018

\begin{abstract}
Biomineralized structures consist of an organic matrix and mineral constituents. Imaging of the mineralized biological tissues is demanding due to specific requirements for the preservation and visualization of chemically different constituents and due to sectioning difficulties. In this study, a characterization of the cuticular matrix of the crustacean exoskeleton was performed by a combination of microscopic methods, aiming to obtain spatial information on the matrix composition. Histochemical procedures were performed and compared in artificially decalcified and non-decalcified samples, in paraffin and resin sections. Wheat germ agglutinin (WGA) lectin-gold conjugate and a fluorescent chitinbinding probe were used to localize chitin in paraffin and resin sections of samples prepared by different fixations. Calcified regions of the matrix were determined by histochemical staining of aldehyde-fixed, methanol-fixed, and resinembedded samples and by scanning electron microscopy with energy dispersive X-ray spectroscopy (SEM-EDXS) of "intact" cuticle transverse profiles that were not subjected to any surface processing. We show that the spatial distribution of cuticular organic matrix components is not homogenous, as a differential staining of exocuticular and endocuticular matrix was obtained by histochemistry. Chitin localization, performed by two different methods, shows a localization pattern of horizontal lines of alternating intensity in the transversely cut endocuticle. Histochemical demonstration of calcified cuticular matrix in resin sections was successful in $2.5 \mu \mathrm{m}$ thick resin sections of methanol-fixed samples. Methanol-fixed, dried, and fractured samples displayed the characteristic elemental composition and are useful to obtain qualitative composition data from the non-processed surface profiles of the cuticle.
\end{abstract}

Keywords: Chitin, calcification, exoskeleton, crustacea, lectin labeling

\section{Introduction}

Biological mineralized structures consist of an organic matrix and minerals. In general, the basic scaffold of the organic matrix consists either of chitin-protein fibers or of collagen molecules, comprising an assemblage of additional macromolecules, including acidic proteins, glycoproteins, and glycosaminoglycans [1]. Exoskeletal cuticle of crustaceans, including isopod crustaceans, is a calcified chitinous matrix $[2,3]$, formed by epidermal cells during animal embryonic and postembryonic development [4-10] and during cuticle replacement in molting adults [11-17]. Mineral content of the cuticle in adult crustaceans is well characterized $[3,18,19]$, whereas data on organic matrix are less complete. Cuticle scaffold consists of chitin-protein fibers, arranged in a characteristic helicoidal pattern [3, 20-22]. In addition to this network, proteins, glycosaminoglycans, and lipids are embedded in the matrix, but the detailed composition and distribution of these macromolecules in the cuticle are not well known. Characterization and localization of the organic matrix constituents at different scale levels are necessary to understand formation, calcification, and renewal of calcified biological structures.

Microscopic characterization of calcified matrices is methodologically challenging, as two key prerequisites have to be fulfilled: chemically diverse sample constituents have to be preserved during all steps, and sample has to be prepared appropriately for imaging, which generally implies that thin sectioning is necessary. Preparation of calcified tissues for sectioning frequently involves artificial decalcification, which could impact also other components in the sample [23-25]. Generally, it is known that crustacean cuticle, once fully calcified, includes different calcium minerals, namely, crystalline calcium carbonate (calcite), amorphous calcium carbonate (ACC), and amorphous calcium phosphate (ACP) [16, 18, 19, 26-30]. Most

\footnotetext{
* Author for correspondence: nada.znidarsic@bf.uni-1j.si
}

phosphorus in the cuticle occurs in ACP [27, 30], and a significant proportion of magnesium occurs within the calcite crystal lattice as magnesium calcite [18, 19, 27].

In this study, different protocols for cuticle matrix characterization at the histochemistry and electron microscopy levels were applied, compared, and adapted, to gain information on the exocuticle and endocuticle matrix composition. The specific goals of this study were as follows:

(a) Evaluation of the cuticular matrix characteristics based on the staining by the selected histological/histochemical procedures, including hematoxylin-eosin, Alcian blue, and Methylene blueAzure II staining; staining results comparison in non-decalcified and ethylenediamine-tetracetic acid (EDTA)-decalcified sections; adaptation of the protocols for application in resin semithin sections;

(b) Assessment of the applicability of the chitin binding probe conjugated to fluorescein isothiocyanate (CBP-FITC) to localize chitin in non-decalcified and EDTA-decalcified paraffin sections and in resin sections of crustacean cuticle.

(c) Comparison of chitin localization by CBP-FITC at the level of fluorescence microscopy and by wheat germ agglutinin (WGA) lectin-gold conjugate at the level of transmission electron microscopy;

(d) Cuticular calcified regions determination in resin sections by histochemistry and in "intact" cuticle surfaces by scanning electron microscopy with energy dispersive X-ray spectroscopy (SEM-EDXS).

\section{Experimental Details}

2.1. Tissue Samples. The tergite cuticle of terrestrial crustacean Porcellio scaber (Isopoda) was analyzed in this study. The thoracic part of the animal body (pereon) consists of seven body segments (pereonites). Tergite is the dorsal exoskeletal part of each pereonite (Figure 1a). In the transverse section of the cuticle, the characteristic horizontal regions are evident, a thin 


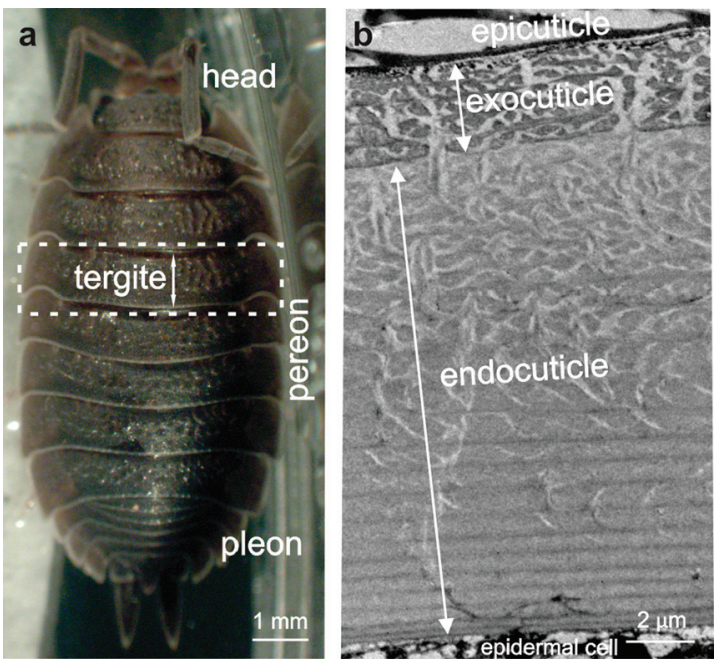

Figure 1. The tergite cuticle of the isopod Porcellio scaber. (a) The body of the animal is divided into three distinct regions: head, pereon, and pleon. Each segment of the pereon is covered by a tergite cuticle. (b) The ultrastructure of the cuticle transverse section shows the organization in three intricate layers: a thin epicuticle on the surface, which also forms prominent scales, exocuticle beneath, and endocuticle facing the epidermal cells

epicuticle on the surface, exocuticle, and endocuticle (Figure 1b). In our study, the animals with no external signs of molting were collected and tergites were isolated and prepared for microscopic imaging as specified in the experimental details for each method below.

2.2. Histochemical Stainings. Two procedures generally used to demonstrate tissue components were used in our study, hematoxylin-eosin staining for paraffin sections and methylene blue-Azure II staining for resin semithin sections. To localize Alcian blue reactive components that include acidic binding sites at the $\mathrm{pH}$ applied, Alcian blue staining was performed in paraffin and in resin sections. Sequential sections were used for different staining procedure to allow comparison.

For paraffin sectioning, the first five tergites were sagittally cut into halves and the corresponding halves were fixed overnight in two different fixatives: one in $3.7 \%$ formaldehyde in $0.1 \mathrm{M}$ Hepes buffer ( $\mathrm{pH} 7.2$ ) and the other in $3.7 \%$ formaldehyde in $0.1 \mathrm{M}$ Hepes buffer (pH 7.2) containing 2.5\% EDTA. After fixation, samples were dehydrated in an ascending series of ethanol and in xylene, infiltrated with paraffin wax (Paraplast Plus, McCormick Scientific) at $60{ }^{\circ} \mathrm{C}$ overnight and embedded afterwards using paraffin embedding station HistoCore Arcadia $\mathrm{H}$ (Leica). Sections $(7 \mu \mathrm{m})$ were cut with a Leica RM2265 microtome, transferred to water on microscope slides, and stretched and dryed on a hot plate.

Resin sections were prepared from isolated tergites fixed in $2 \%$ paraformaldehyde and $2.5 \%$ glutaraldehyde in $0.1 \mathrm{M}$ cacodylate buffer ( $\mathrm{pH}$ 7.2). The samples were washed in buffer, dehydrated and embedded in Agar 100 epoxy resin, and the $0.5 \mu \mathrm{m}$ thick sections were prepared with a glass knife using a Reichert Ultracut S ultramicrotome (Leica).

Hematoxylin-eosin staining of paraffin sections was performed in the standard procedure as described in Kiernan [25] and Bancroft and Gamble [31], using Weigert's iron hematoxylin. Methylene blue-Azure II staining was applied to resin sections in the standard procedure described in Robinson et al. [32]. Alcian blue staining was performed in 1\% Alcian blue solution in 3\% acetic acid $(\mathrm{pH} 2.5)$. Pre-staining preparation of the resin sections included immersion in $3 \% \mathrm{NaOH}$ in absolute ethanol for approximatelly $5 \mathrm{~min}$ and then in buffer to remove the resin. The duration of staining was $5 \mathrm{~min}$ for paraffin sections and $2 \mathrm{~h}$ for resin sections, and afterwards, they were quickly washed in 3\% acetic acid and rinsed in deionized water. Counterstaining of paraffin sections was performed by Kernechtrot ( $5 \mathrm{~min}$ ), followed by dehydration and mounting in Pertex. Resin sections were finally embedded in Ultrakitt. Imaging was performed with a Zeiss AxioImager Z.1 light microscope, equipped with an HRC Axiocam camera and Axiovision software.

2.3. Labeling with WGA Lectin and Transmission Electron Microscopy. Tergites were isolated, and tergite pieces were immediately immersed in fixative containing $2 \%$ paraformaldehyde, $0.25 \%$ glutaraldehyde, and $2.5 \%$ EDTA in $0.1 \mathrm{M}$ Hepes buffer ( $\mathrm{pH}$ 7.2). Samples were dehydrated and embedded in London Resin (LR) white resin, polymerized by accelerator in ice bath. Ultrathin sections were made by Ultracut S (Leica) set to nominal section thickness of $70 \mathrm{~nm}$. Labeling with lectin wheat germ (Triticum vulgare) agglutinin was performed on ultrathin sections. Ultrathin sections were incubated in $1 \%$ bovine serum albumin (BSA, Sigma) in $0.1 \mathrm{M}$ Hepes for $5 \mathrm{~min}$, followed by incubation in WGA conjugated to $10 \mathrm{~nm}$ gold (Gentaur) for $1 \mathrm{~h}$ at room temperature. After washing in $0.1 \mathrm{M}$ Hepes, the sections were fixed in $1.25 \%$ glutaraldehyde in $0.1 \mathrm{M}$ Hepes for $30 \mathrm{~min}$ at room temperature and washed in $0.1 \mathrm{M}$ Hepes and in deionized water. Other tissues in the section, e.g., muscles, were considered as negative controls. Sections were inspected with a Philips CM100 transmission electron microscope, equipped with BioScan 792 and Orius 200 (Gatan) cameras, and imaged using Digital Micrograph software.

2.4. Chitin Localization by a Fluorescein-Conjugated Chitin-Binding Probe. Chitin-binding probe is a protein that binds specifically to chitin, as it comprises a chitin-binding domain, derived from chitinase. Labeling with the CBP-FITC (New England BioLabs Inc.) was applied to paraffin- and resinembedded sections. Specimens for paraffin sections were processed as described in Section 2.2. (Histochemical stainings), using both fixation procedures: one half of the tergite was fixed without EDTA and the other half with EDTA (artificial decalcification). Specimens for resin sections were processed in two different ways: (1) overnight fixation in $2 \%$ paraformaldehyde, $2.5 \%$ glutaraldehyde, and $2.5 \%$ EDTA in $0.1 \mathrm{M}$ Hepes buffer ( $\mathrm{pH} 7.2$ ), postfixation in $1 \%$ osmium tetroxide $(1 \mathrm{~h})$, dehydration in ethanol and in acetone, and embedding in Agar 100 epoxy resin; (2) overnight fixation in absolute methanol and direct embedding in Agar 100 epoxy resin.

Labeling with CBP-FITC was performed on $7 \mu \mathrm{m}$ paraffin sections and on $0.5 \mu \mathrm{m}$ semithin sections, prepared as described in Section 2.2. (Histochemical stainings). Before CBP-FITC incubation, the paraffin sections were deparaffinized and rehydrated to distilled water. The resin sections were immersed in $3 \% \mathrm{NaOH}$ in absolute ethanol for approximatelly $5 \mathrm{~min}$ and then in buffer to remove the resin. All sections were incubated in CBP-FITC, diluted 1:500 in 0.1 M Hepes buffer ( $\mathrm{pH} 7.2$ ), overnight at room temperature in dark, and then washed three times for $7 \mathrm{~min}$ in buffer. Simultaneously, the sections for negative control were incubated overnight in buffer. Following CBP-FITC incubation and washing, the sections were mounted in Fluoroshield with DAPI (4',6-Diamidine-2'-phenylindole dihydrochloride) (Sigma-Aldrich). Sections were imaged with a Zeiss AxioImager Z.1 light microscope, in differential interference contrast (DIC) mode and in flourescence mode using Filter set 01 for DAPI (excitation BP 365/12, beam splitter FT 395, emission LP 397) and Filter set 09 for FITC (excitation BP 450-490, beam splitter FT 510, emission LP 515). Merging of the images was performed using software ImageJ.

\subsection{Calcified Matrix Localization}

2.5.1. Histochemical Alizarin Red S Staining. Alizarin red $\mathrm{S}$ (ARS) staining, a histochemical method for calcified tissue 
demonstration, was applied to resin sections. The isolated tergites, sagittally cut in two halves were fixed overnight either in fixative containing $2 \%$ paraformaldehyde and $2.5 \%$ glutaraldehyde in $0.1 \mathrm{M}$ cacodylate buffer $(\mathrm{pH} \mathrm{7.2)}$ or in absolute methanol. The aldehyde-fixed samples were then washed in buffer, dehydrated, and embedded in Agar 100 resin, and the methanol-fixed samples were directly embedded in resin. Semithin sections of $0.5 \mu \mathrm{m}$ and $2.5 \mu \mathrm{m}$ thickness were prepared from all samples and transferred into water drop on glass slide. After drying, they were stained with $0.3 \%$ ARS staining solution in $80 \%$ ethanol for $2 \mathrm{~h}$ at room temperature, then washed in $80 \%$ ethanol, dried, mounted in Ultrakitt, and imaged.

2.5.2. Energy Dispersive X-ray Spectroscopy in FEG-SEM. To determine the elemental composition of the cuticle in the tergites of adult animals, we have performed the EDXS in a field-emission gun scanning electron microscope (FEGSEM). Tergites were cut sagittally into two halves and fixed in absolute methanol (2 days) to retain also amorphous calcium minerals. Sample fixation by methanol was suggested to preserve also amorphous calcium minerals within the cuticle [47]. The samples were then air-dried, fractured to expose internal transverse profiles of the cuticle, mounted to specimen stubs using silver paste (SPI Supplies), and coated with approximately $5 \mathrm{~nm}$ carbon layer. EDXS analyses were performed in a high-resolution field-emission gun scanning electron microscope (JEOL JSM-7600F) equipped with an Oxford Instruments INCA system for energy dispersive X-ray spectroscopy. Imaging and EDXS measurements were performed at acceleration voltage of $15 \mathrm{kV}$ and a working distance of $15 \mathrm{~mm}$. Each X-ray spectrum was collected for $180 \mathrm{~s}$ using INCA software.

\section{Results and Discussion}

3.1. Histochemical Stainings. In hematoxylin-eosin staining, a clear distinction between the exocuticle and endocuticle composition is evidenced; exocuticle is strongly stained by hematoxylin and endocuticle by eosin (Figure 2a and c). In decalcified samples, this difference is even more pronounced, while in non-decalcified samples the endocuticle is also slightly stained bluish due to hematoxylin. This suggests that exocuticular matrix is rich in acidic (basophilic) binding sites, which are generally stained by hematoxylin, and that endocuticular matrix is characterized primarily by basic (acidophilic) binding sites, commonly stained by eosin, at the staining conditions applied. In the Alcian blue staining, the cuticle is clearly differentially stained blue in comparison to subjacent tissues that are counterstained pink (Figure $2 b$ and $d$ ). This reveals abundance of Alcian blue reactive substances in the cuticular matrix at the $\mathrm{pH}$ applied $(\mathrm{pH} 2.5)$, i.e., acidic molecules. Artificial decalcification apparently did not influence Alcian blue staining considerably. Alcian blue staining applied to epoxy resin sections requires a preceding removal of the resin by $\mathrm{NaOH}-$ absolute ethanol solution, as suggested by Litwin and Kasprzyk [33]. Staining of resin embedded cuticle samples with preceding resin removal and prolonged staining duration results in Alcian blue positive exocuticle only, while endocuticle is not stained (Figure 2f). Methylene blue-Azure II staining that is commonly used for semithin resin sections results in a differential dark blue exocuticle staining in comparison to light blue endocuticle (Figure 2e). In addition, non-homogeneous endocuticle staining was evident in certain samples, with basal layers stained more intense in comparison to the rest of the endocuticle. In summary, we show that exocuticle and endocuticle differ in the composition of the organic matrix. Our results complement data on differences between the mineral composition of exocuticle and endocuticle reported by Hild et al. [18]. Hild et al. [18] established that calcite is present only in the exocuticle and the endocuticle contains only amorphous minerals.
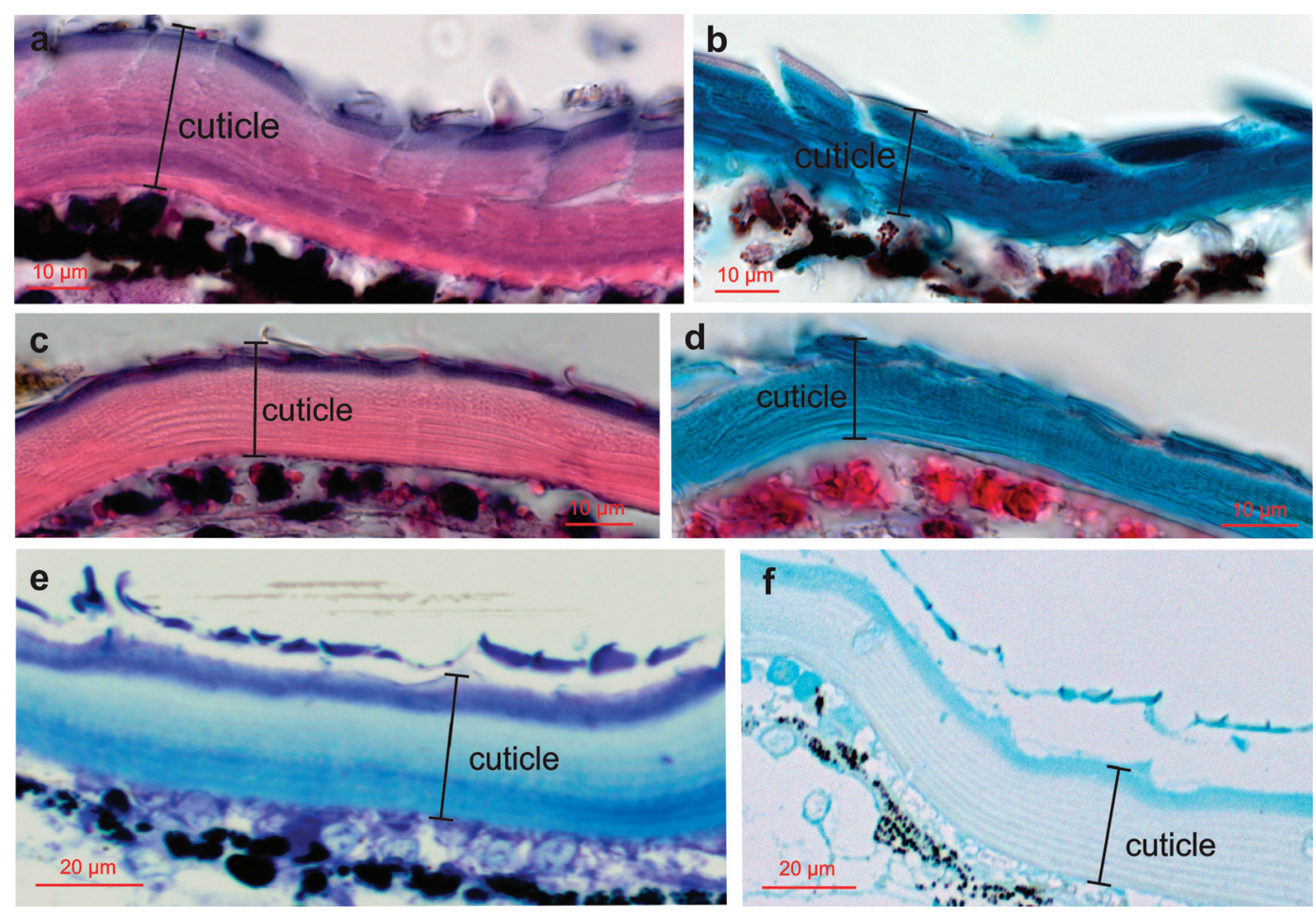

Figure 2. Histochemical stainings of $P$. scaber tergite cuticle, applied to paraffin sections (a-d) of non-decalcified samples (a, b) and decalcified sample (c, d), and to resin sections (e, f). a and c: Hematoxylin-eosin staining. The exocuticle is strongly stained blue by hematoxylin and the endocuticle pink by eosin. In non-decalcified sample, the endocuticle is slightly hematoxylin positive. $\mathrm{b}$ and d: Alcian blue staining shows differentially stained cuticle (blue) in all samples. e: In methylene blue-Azure II method, the exocuticle is stained dark blue and the endocuticle light blue. f: Alcian blue staining shows stronger reaction to exocuticle in comparison to only background stained endocuticle 


\subsection{Labeling with WGA Lectin and Transmission} Electron Microscopy. Localization of $N$-acetyl-glucosamine oligomers, including chitin, was performed by WGA lectin-gold labeling. Exocuticle and endocuticle were both intensely labeled (Figure 3), while the underlaying tissues were not. The results show that labeling in the endocuticle is not homogenous, but labeling density appears to alternate in horizontal layers (Figure $3 \mathrm{e}$ and $\mathrm{f}$ ). This labeling pattern could be explained by a non-homogeneous distribution of WGA-binding ligands in the endocuticle or by a variation of their binding sites accessibility. WGA lectin has frequently been used as a chitin-localization probe [34-36], as it binds highly specifically to $N$-acetyl-Dglucosamine and has a strong affinity to oligomers and polymers of $N$-acetyl-glucosamine, especially to chitin [37, 38]. The combining site of wheat germ agglutinin was reported to be complementary to a sequence of three $\beta$-(1/4)-linked $N$-acetylglucosamine residues [39], but interactions of WGA lectin with $\mathrm{N}$-acetylneuraminic acid containing glycoconjugates [40] and poly- $N$-acetyllactosaminoglycans [41] were also reported. Therefore, WGA binding needs to be interpreted with caution in the case of chitin localization and additional complementary analyses are advantageous [7, 42-45]. In our study, chitin localization by a fluorescein-conjugated chitin-binding probe was applied to further clarify this issue.

3.3. Chitin Localization by a Fluorescein-Conjugated Chitin-Binding Probe. We show that a fluorescein-conjugated chitin-binding probe (New England BioLabs Inc.) is successfully applied for chitin localization in paraffin sections of the crustacean cuticle that was artificially decalcified by EDTA as well in the samples that were not subjected to decalcification procedure (Figure 4). A clear chitin labeling is evidenced in the cuticle transverse sections, and a pattern of alternating horizontal lines of higher and lower intensities is revealed in all samples.

Next, we show the applicability of this procedure to demonstrate chitin distribution in resin sections of the crustacean cuticle fixed in aldehydes, as well as fixed in methanol (Figure 5). Alternating lines of higher and lower fluorescence intensities are evidenced also in resin semithin sections (Figure $5 \mathrm{~d}-\mathrm{h}$ ). It is clearly shown that this pattern is revealed in the endocuticle. Interestingly, Hild et al. [18] reported a similar alternating lines pattern in the Raman imaging of organic constituents
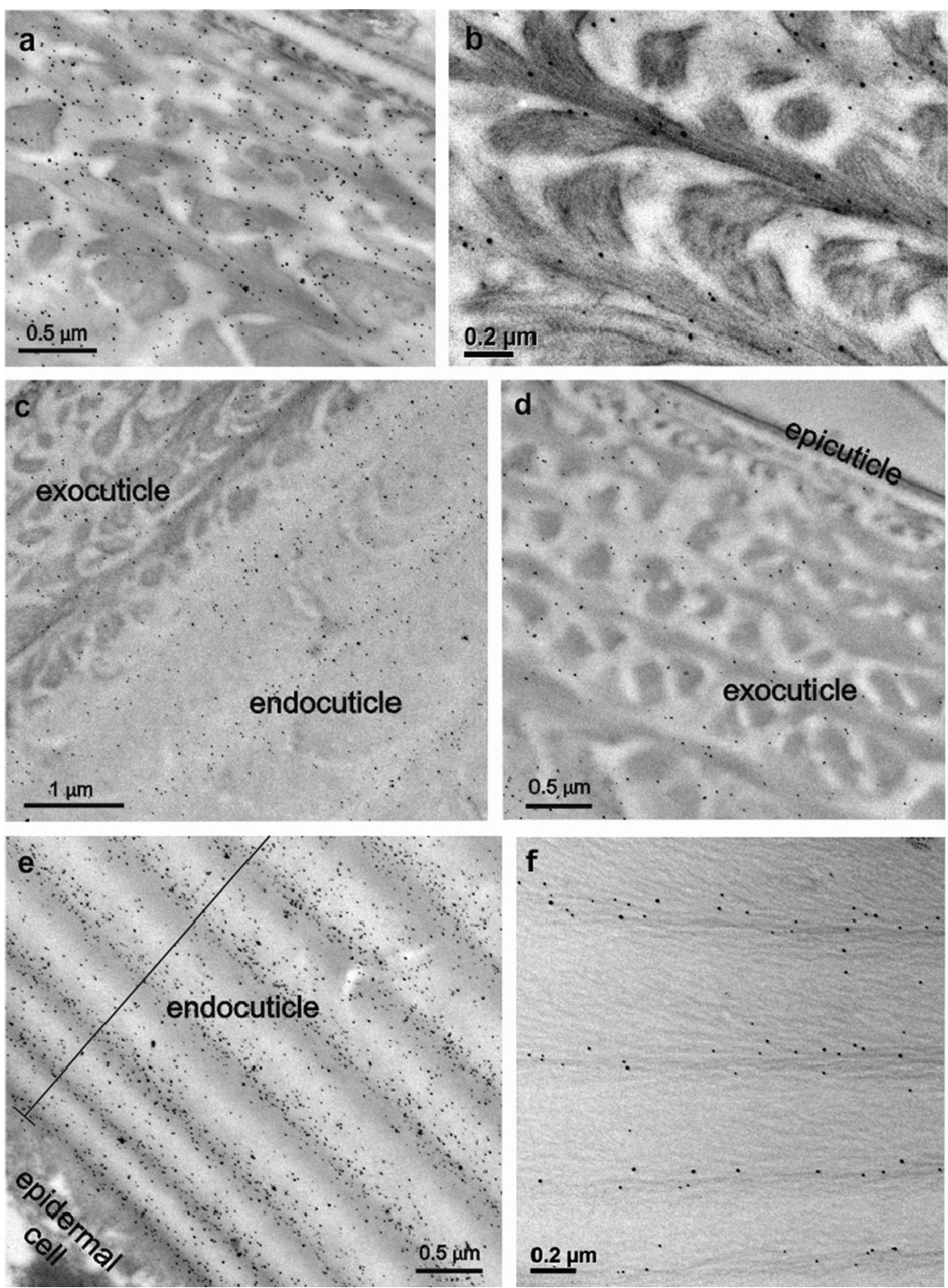

Figure 3. Transmission electron microscopy images of the localization of $N$-acetyl-glucosamine oligomers, including chitin, by the WGA lectingold labeling. Images show localization reaction in different regions of the cuticle, exocuticle $(a-d)$ and endocuticle (e, f). Ultrathin sections shown were contrasted, except the section in (d) that was not stained after localization reaction 

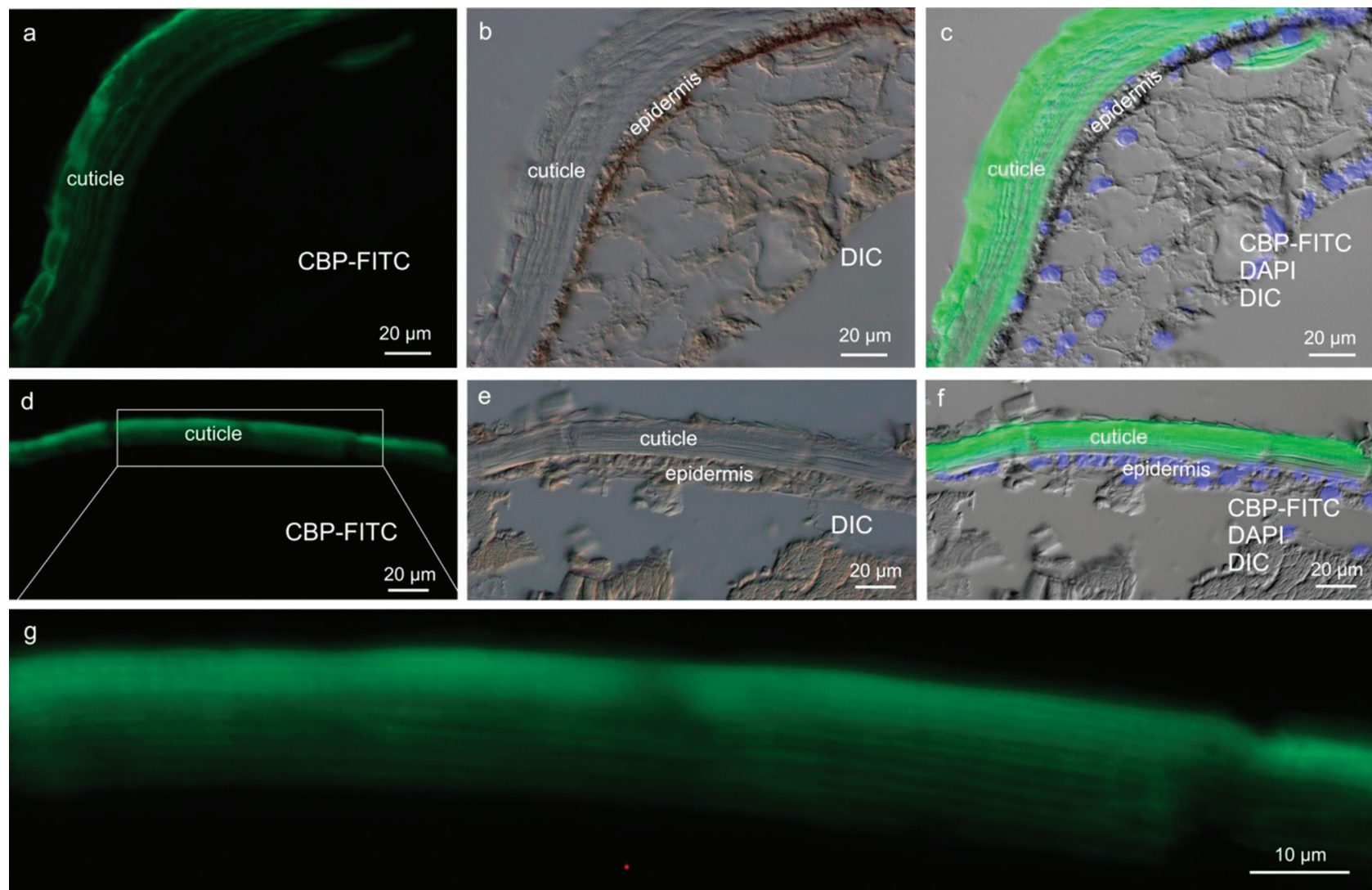

Figure 4. Labeling of $P$. scaber tergite cuticle with a chitin-binding probe conjugated to FITC, applied to paraffin sections. Fluorescence images of tergite labeled with CBP-FITC (a, d, g). DIC images (b, e) taken at the same position as images a and d, respectively. Merged images (c and f): from images CBP-FITC, DIC, and DAPI-stained cell nuclei. Figures a, b, and c show a non-decalcified sample, and figures d, e, f, and g show a decalcified sample

in the cuticle of the same species. The labeling pattern in the exocuticle cannot be unambiguously classified as homogenous or non-homogenous. In aldehyde-fixed samples, better quality sections could be obtained and CBP-FITC imaging indicates that exocuticle labeling is less intense in comparison to endocuticle. The thin epicuticular layer on the surface was not labeled by the chitin probe. This is clearly revealed in Figure 5h, where it is visible that the superficial thin epicuticular layer is slightly detached due to sectioning and is not stained. This is in concert with epicuticle composition in isopods reported before, consisting primarily of lipids, proteins, and lipoproteins that are organized in two main sublayers, the outer epicuticle and the inner epicuticle $[12,14,46]$.

3.4. Calcified Matrix Localization. Calcification is one of the key characteristics of the chitinous matrix in the crustacean exoskeletal cuticle, and a spectrum of methods is available to analyze this issue, differing in sample preparation demands, resolution, detection limits, the type of information obtained, and in cost/time input. We present here a histochemical calcified tissue localization, a relatively simple and quick procedure to identify calcified matrix at the level of light microscopy, and calcium localization by energy dispersive X-ray spectroscopy at the level of electron microscopy in the sample previously prepared by a very simple and quick approach, aimed primarily to omit any sample surface processing. The samples for histochemical staining with Alizarin $\mathrm{S}$ were prepared by two different procedures, fixed in aldehydes or in methanol and resinembedded, while samples for EDXS were methanol-fixed and dried only. Methanol fixation of the samples and no exposure to water during sample preparation were performed in order to preserve also amorphous calcium minerals within the cuticle, as suggested in Becker et al. [47] and as high solubility of ACC in aqueous solutions was reported in Brečević and Nielsen [48], Gal et al. [49], and Meiron et al. [50].

3.4.1. Histochemical Alizarin Red S Staining. Histochemical method ARS is usually applied to paraffin and cryostat sections, which are considerably thicker, while less studies are available that demonstrate calcified tissue by ARS staining in epoxy resin sections. Some data on ARS staining of vertebrate samples are available, e.g., bone tissue culture [51], bone [52, 53], and atherosclerotic plaques [54], using aldehyde fixation of the samples and aqueous solution of ARS. Here, we show the results of epoxy sections staining by ethanol solution of ARS, without preceding resin removal. A positive histochemical reaction of ARS was evident in the external part of the cuticle in aldehyde-fixed and in methanol-fixed samples, embedded in resin (Figure 6). Only in methanol-fixed thick resin sections $(2.5 \mu \mathrm{m})$ the whole transverse profile of the cuticle was intensely stained (Figure 6d), with an exception of a few inner lamellae flanking the subjacent epidermal cells evident in certain regions. These results show that sample fixation and section thickness impact histochemical calcified matrix localization by ARS ethanol solution in resin sections. As it is known that exocuticle in adult animals contains calcite, whereas the mineral phase in the inner cuticle layers (endocuticle) is amorphous [18], we consider that amorphous forms of calcium minerals were preserved and stained only in the thick sections of methanolfixed/resin-embedded cuticle. In all other cuticle samples, the calcium compounds in the endocuticle were lost during procedure. In the case of methanol-fixed/resin-embedded samples, we consider that loss was due to material extraction 

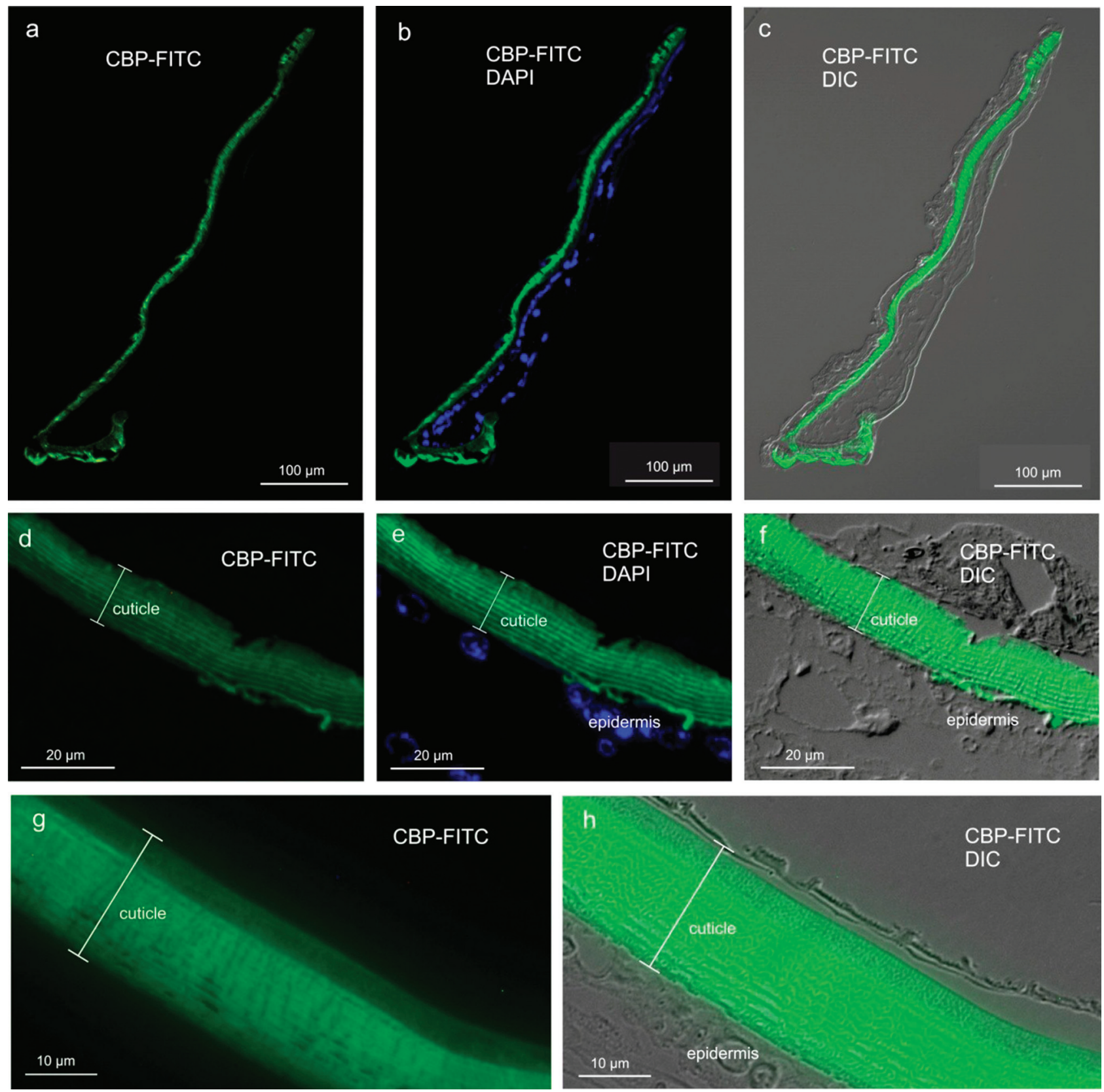

Figure 5. Labeling of $P$. scaber tergite cuticle with a chitin-binding probe conjugated to FITC, applied to resin sections of the methanol-fixed samples (a-f) and aldehyde-fixed and decalcified samples (g, h). Fluorescence images of tergites labeled with CBP-FITC (a, d, g). Merged images ( $b$ and e): from CBP-FITC images and cell nuclei images labeled with DAPI. Merged images (c, f, and h): from CBP-FITC images and DIC

from the $0.5 \mu \mathrm{m}$ sections floating on the water drop on the slide.

3.4.2. Energy Dispersive X-ray Spectroscopy in FEG-SEM. EDXS spectra of the transverse profiles of methanol-fixed samples show that calcium is retained and detected in both, in the exocuticle and in the endocuticle of the tergite. The results show prominent contents of calcium, with the evidently high ratio of calcium to phosphorus peaks (Figure 7). Besides calcium and phosphorus, principal elements detected in the cuticle of adults were also sulfur, magnesium, oxygen, and carbon. Our results are in concert with the elemental composition of the tergite cuticle recorded before in different isopod species, obtained by atomic absorption spectroscopy [27] and by EDXS of the samples that were subjected in addition to polishing with a diamond knife $[18,19,29,55]$. We show that a simplified preparation used in our study is useful as a quick non-quantitative approach to obtain information on elemental compositon of intact sample (no sectioning or polishing applied) and at spatial resolution adequate to distinguish different regions within the cuticle.

\section{Conclusions}

- We show that the spatial distribution of cuticular organic matrix components is not homogenous, as a differential staining of exocuticular and endocuticular matrix is obtained by histochemical procedures applied to paraffin and resin sections. Artificial decalcification by EDTA influences hematoxylineosin staining but does not have a major impact on Alcian blue staining.

- Chitin localizations performed by two different methods, WGA lectin-gold conjugate and CBP-FITC fluorescent probe, both show a localization pattern of horizontal lines 

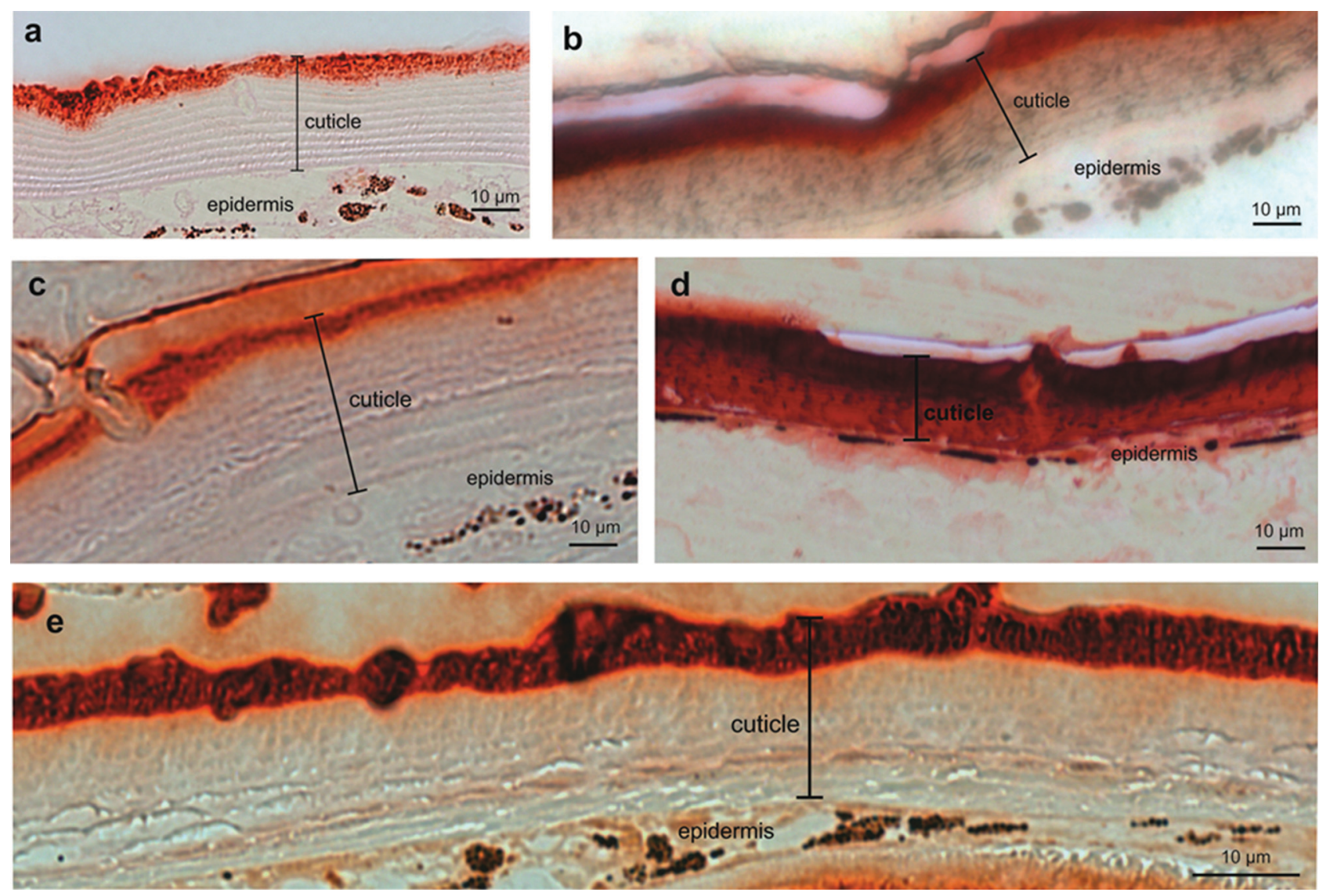

Figure 6. ARS staining of $P$. scaber tergite cuticle, applied on resin sections of: (a, b) aldehyde-fixed sample and (c-e) methanol-fixed sample. In the $0.5 \mu \mathrm{m}$ thick sections of all samples (a, c, e) and in the $2.5 \mu \mathrm{m}$ thick sections of aldehyde-fixed sample (b), a positive reaction is evident in exocuticle, while the endocuticle is not intensely stained. (d) In the $2.5 \mu \mathrm{m}$ thick section of methanol-fixed samples, the whole transversal profile of the cuticle is intensely stained
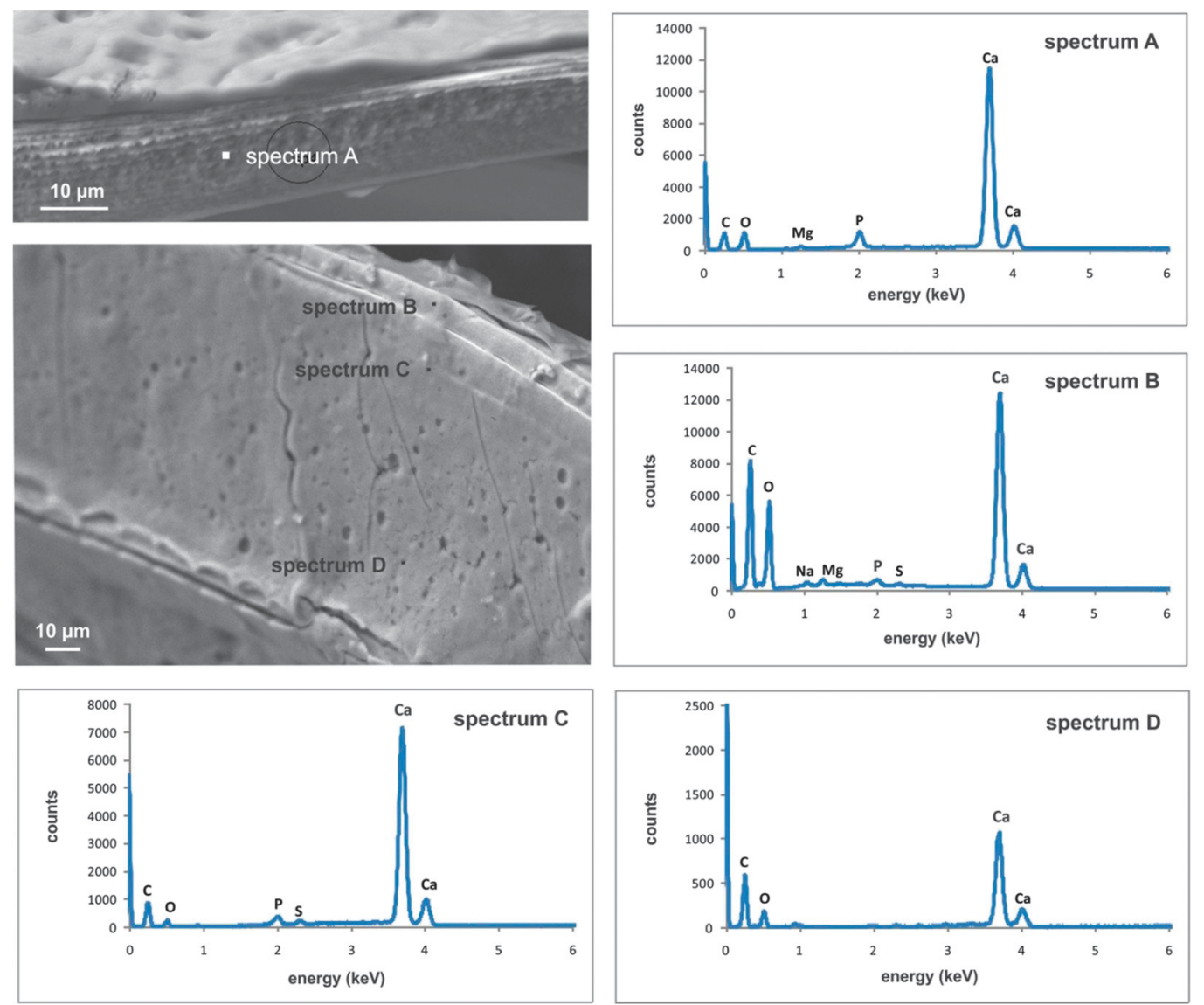

Figure 7. EDXS spectra, obtained from the P. scaber tergite cuticle in different regions of the cuticle transversal fracture. All spectra show prominent contents of calcium and high ratio of calcium to phosphorus peaks. Sulfur, magnesium, oxygen, and carbon peaks are also evident 
of alternating variation in intensity within the transversely cut endocuticle. This suggests that either macromolecules that bind to the probes used are distributed in a non-homogeneous arrangement or that the observed pattern is due to variation of their binding sites accessibility.

- Histochemical Alizarin red S (0.3\% solution in 80\% ethanol) demonstration of calcified cuticular matrix in resin sections was successful in methanol-fixed, resin-embedded sample sections of $2.5 \mu \mathrm{m}$ in thickness. Thus, we suggest applying this procedure in further studies on resin-embedded cuticle. Calcified matrix of the endocuticle was not detected in $0.5 \mu \mathrm{m}$ sections of methanol-fixed samples and in $0.5 \mu \mathrm{m}$ or $2.5 \mu \mathrm{m}$ sections of aldehyde-fixed samples.

- Cuticle samples prepared by methanol fixation, drying, and transverse fracturing displayed the characteristic elemental composition and are thus useful to obtain qualitative data on the composition from the non-processed surface profile of the cuticle.

Acknowledgments. The research was financially supported by Slovenian Research Agency, project P1-0184. We acknowledge Urban Bogataj for image processing by ImageJ.

Open Access. This is an open-access article distributed under the terms of the Creative Commons Attribution-NonCommercial 4.0 International License (https://creativecommons.org/licenses/ by-nc/4.0/), which permits unrestricted use, distribution, and reproduction in any medium for non-commercial purposes, provided the original author and source are credited, a link to the CC License is provided, and changes - if any - are indicated.

\section{References}

1. Ehrlich, H. Int. Geol. Rev. 2010, 52, 661-699.

2. Compere, P.; Jeuniaux, C.; Goffinet, G. The Integument: Morphology and Biochemistry, in The Crustacea vol. 1, Forest, J.; von Vaupel Klein, J. C.; Schram, F. R. eds., Brill, Leiden, 2004, pp. 59-144.

3. Dillaman, R.; Roer, R.; Shafer, T.; Modla, S. The Crustacean Integument: Structure and Function, in Functional Morphology and Diversity, Watling, L.; Thiel, M. eds., Oxford University Press, New York, 2013, pp. 140-166.

4. Goudeau, M. J. Morphol. 1976, 148, 427-452.

5. Glas, P. S.; Courtney, L. A.; Rayburn, J. R.; Fisher, W. S. Biol. Bull. 1997, 192, 231-242.

6. Rosowski, J. R.; Belk, D.; Gouthro, M. A.; Lee, K. W. J. Shellfish Res. 1997, 16, 233-249

7. Havemann, J.; Müller, U.; Berger, J.; Schwarz, H.; Gerberding, M.; Moussian, B. Cell Tissue Res. 2008, 332, 359-370.

8. Mrak, P.; Žnidaršič, N.; Tušek-Žnidarič, M.; Klepal, W.; Gruber, D.; Štrus, J. Zookeys. 2012, 176, 55-72.

9. Mrak, P.; Žnidaršič, N.; Žagar, K.; Čeh, M.; Štrus, J. Arthropod Struct. Dev. 2014, 43, 423-439.

10. Mrak, P.; Bogataj, U.; Štrus, J.; Žnidaršič, N. Arthropod Struct. Dev. 2017, 46, 77-95.

11. Price, J. B.; Holdich, D. M. Zoomorphology. 1980, 95, 250-263.

12. Compere, P. Biology of Terrestrial Isopods, Proceedings of the Third International Symposium on the Biology of Terrestrial Isopods, Poitiers, July $10-12,1990,169-175$.

13. Štrus, J.; Compere, P. Eur. J. Physiol. 1996, 431, R251-R252.

14. Ziegler, A. Tissue Cell. 1997, 29, 63-76.

15. Štrus, J.; Blejec, A. Isopod Systematics and Evolution. Crustacean Issues, in Kensley, B.; Brusca, R. C. eds., 2001, 13, 343-352.
16. Dillaman, R.; Hequembourg, S.; Gay, M. J. Morphol. 2005, 263, $356-374$.

17. Vittori, M.; Kostanjšek, R.; Žnidaršič, N.; Štrus, J. Zookeys. 2012, 176 23-38.

18. Hild, S.; Marti, O.; Ziegler, A. J. Struct. Biol. 2008, 163, 100-108.

19. Hild, S.; Neues, F.; Znidaršič, N.; Štrus, J.; Epple, M.; Marti, O.; Ziegler, A. J. Struct. Biol. 2009, 168, 426-436.

20. Giraud-Guille, M. M. Curr. Opin. Solid State Mater. Sci. 1998, 3, $221-227$.

21. Raabe, D.; Romano, P.; Sachs, C.; Fabritius, H.; Al-Sawalmih, A.; Yi, S.-B.; Servos, G.; Hartwig, H. G. J. Mater. Sci. Eng. A. 2006, 421, 143-153.

22. Fabritius, H.; Sachs, C.; Romano Triguero, P.; Raabe, D. Adv. Mater. 2009, 21, 391-400.

23. Hosoya, A.; Hoshi, K.; Sahara, N.; Ninomiya, T.; Akahane, S.; Kawamoto, T.; Ozawa, H. Histochem Cell Biol. 2005, 123, 639-646.

24. Bonucci, E. Methodology. in Biological calcification: Normal and Pathological Processes in the Early Stages, Schreck, S. ed., Springer-Verlag, Heidelberg, 2007, pp. 23-51.

25. Kiernan, J. A. Histological and Histochemical Methods: Theory and Practice, 4th ed., Scion Publishing Limited, Bloxham, 2008, 45-51 and 141-157.

26. Roer, R.; Dillaman, R. Am. Zool. 1984. 24, 893-909.

27. Becker, A.; Ziegler, A.; Epple, M. Dalton Trans. 2005, 10, 1814-1820.

28. Neues, F.; Hild, S.; Epple, M.; Marti, O.; Ziegler, A. J. Struct. Biol. 2011, $175,10-20$.

29. Seidl, B.; Huemer, K.; Neues, F.; Hild, S.; Epple, M.; Ziegler, A. J. Struct. Biol. 2011, 174, 512-526.

30. Luquet, G. Zookeys. 2012, 176, 103-121.

31. Bancroft, J. H.; Gamble, M. Theory and Practice of Histological Techniques, 6th ed., Elsevier, Churchill Livingstone, 2008, pp. 121-134.

32. Robinson, D. G.; Ehlers, U.; Herken, R.; Herrmann, B.; Mayer, F.; Schürmann, F.-W. Methods of Preparation for Electron Microscopy, An Introduction for the Biomedical Sciences, Springer-Verlag, Berlin, Heidelberg, 1987, 190

33. Litwin, J. A.; Kasprzyk, J. M. Acta Histochem. 1980, 67, 265-271.

34. Shillito, B.; Lübbering, B.; Lechaire, J. P.; Childress, J. J.; Gaill, F. J. Struct. Biol. 1995, 114, 67-75.

35. Neuhaus, B.; Bresciani, J.; Peters, W. Acta Zoologica. 1997, 78, 205-213.

36. Lemburg, C. Zoomorphology. 1998, 118, 137-158.

37. Allen, A. K.; Neuberger, A.; Sharon, N. Biochemical J. 1973, 131, $155-162$.

38. Peters, W.; Latka, I. Histochemistry. 1986, 84, 155-160.

39. Debray, H.; Decout, D.; Strecker, G.; Spik, G.; Montreuil, J. FEBS J. 1981, 117, 41-55.

40. Monsigny, M.; Roche, A. C.; Sene, C.; Maget-Dana, R.; Delmotte, F.

FEBS J. 1980, 104, 147-153.

41. Gallagher, J. T.; Morris, A.; Dexter, M. Biochem. J. 1985, 231, 115-122.

42. Schmidt, J.; Kleffmann, T.; Schaub, G. A. Parasitol. Res. 1998, 84, 527-536.

43. Steinbrecht, R. A.; Stankiewicz, B. A. J. Insect Physiol. 1999, 45, 785-790.

44. Martin, G. G.; Simcox, R.; Nguyen, A.; Chilingaryan, A. Biol. Bull. 2006, 211, 275-285.

45. Moussian, B.; Seifarth, C.; Müller, U.; Berger, J.; Schwarz, H. Arthropod Struct. Dev. 2006, 35, 137-152.

46. Price, J. B.; Holdich, D. M. Zoomorphology. 1980, 94, 321-332.

47. Becker, A.; Bismayer, U.; Epple, M.; Fabritius, H.; Hasse, B.; Shi, J.; Ziegler, A. Dalton Trans. 2003, 4, 551-555.

48. Brečević, L.; Nielsen, A. E. J. Cryst. Growth. 1989, 98, 504-510.

49. Gal, J.-Y.; Bollinger, J.-C.; Tolosa, H.; Gache, N. Talanta. 1996, 43 , 1497-1509.

50. Meiron, O. E.; Bar-David, E.; Aflalo, E. D.; Shechter, A.; Stepensky, D.; Berman, A.; Sagi, A. J. Bone Miner. Res. 2011, 26, 364-372.

51. Gilmore, S. K.; Whitson, S. W.; Bowers Jr., D. E. Stain Tech. 1986, 61, 89-92.

52. Clarke Anderson, H.; Sipe, J. B.; Hessle, L.; Dhamyamraju, R.; Atti, E.; Camacho, N. P.; Millán, J. L. Am. J. Pathol. 2004, 164, 841-847.

53. Clarke Anderson, H.; Harmey, D.; Camacho, N. P.; Garimella, R.; Sipe, J. B.; Tague, S.; Bi, X.; Johnson, K.; Terkeltaub, R.; Millán, J. L. Am. J. Pathol. 2005, 166, 1711-1720.

54. Relucenti, M.; Heyn, R.; Petruzziello, L.; Pugliese, G.; Taurino, M.; Familiari, G. Eur. J. Histochem. 2010, 54, 143-147.

55. Ruangchai, S.; Reisecker, C.; Hild, S.; Ziegler, A. J. Struct. Biol. 2013, $182,22-35$. 